\title{
THEORY OF THE USE OF ARTERIOVENOLS CONCENTRATION DIFFERENCES FOR MEASURING METABOLISM IN STEADY AND NON-STEADY STATES *
}

\author{
BY KEN.NETH I.. ZIERLER
}

(From the Department of Medicine, The Johns Hopkins L'niversity and Hospital, Baltimore, IVd.)

(Submitted for publication July 14, 1961; accepted August 17, 1961)

In increasing numbers investigators are measuring concentrations of certain substances in arterial and in venous blood and equating arteriovenous $(\mathrm{A}-\mathrm{V})$ concentration differences obtained from simultaneous arterial and venous samples to uptake or release of the metabolite by a tissue drained by the venous system sampled. Conditions under which $\mathrm{A}-\mathrm{V}$ concentration differences are valid estimates of tissue metabolism are sharply limited and, although the literature does contain ample warning (e.g., References 1-3), these limitations seem to have been misunderstood so often that it was thought a re-examination of the problem might be helfpul.

Fundamentally, all such investigations wish to employ the Fick principle (4) which states that if : a) arterial concentration of substance $x$ is constant, $b$ ) if venous concentration of $x$ is constant, $c$ ) if blood flow is constant and $d$ ) if uptake (or release) of $x$ is constant, then, input of $x$ (= flow multiplied by arterial concentration $)=$ output of $x$ (= flow multiplied by venous concentration) + tissue metabolism of $x$ (where the tissue metabolism term is positive if there is tissue uptake and negative if there is tissue release).

Fick, of course, proposed this equation as a means of measuring blood flow. The equation can be and is used to find any single characteristic whenever all the others are measured independently, and it is our purpose to consider only. its application to estimation of tissue metabolism.

It is obvious immediatel that $\mathrm{A}-\mathrm{V}$ concentrition differences cannot be equated to tissue uptake (or rclease) unless flow is constant and known. It is correct to speak of changes in

* These studies were aided by Contract Nonr-248(34) (NR 101-241) between the Office of Naval Research, Department of the Navy and The Johns Hopkins University, by a grant-in-aid from the Muscular Dystrophy Associations of America, Inc., and by Public Health Service Research Grant A-750 from the National Institute of Arthritis and Metabolic Diseases. Reproduction in whole or in part is permitted for any purpose of the United States Government. steady state $\mathrm{A}-\mathrm{V}$ differences (i.e., the difference between one steady state and another steady state) as proportional to changes in tissue uptake (or release) if it is known that flow, although not quantified, has not changed. The curious practice has arisen of recognizing that flow must be included in the calculation but then ignoring it on the assumption that it is invariant. Those experienced in measurement of blood flow are familiar with its lability. Blood flow through the forearm of man may increase tenfold, for example, with exercise (5). When circulation to the hand is added to that of the forearm, flow through the brachial artery is easily. doubled and, owing largely to the thermoregulatory function of blood flow to the hand, becomes unstable in the absence of careful control of ambient temperature and humidity. It is, therefore, not at all plausible to dismiss blood flow with the assumption that it was constant because the investigator was unaware that he had done anything to change it. Jiggling an arterial needle during sampling provokes vasodilatation, for example, so that most investigators who have not taken pains to lead blood from an artery through flexible tubing may have altered blood flow by as much as 50 per cent and perhaps even by more than twoofold (observations by R. Andres, G. (ader and K. L. Zierler).

Because blood flow may not be constant we must inquire whether or not we can salvage application of the Fick principle by $a$ ) defining those variations in blood flow that are sufficiently. small to permit use of the Fick principle (that is, if blood flow is treated as invariant, how much can it vary and produce no more than some given acceptable upper bound on the error estimate?), and $b$ ) redefining the Fick principle to include describable unsteady states of blood How.

Validity of the use of the Fick principle depends not only on steady blood flow but also on constant arterial concentration and on constant tissue metabolism. It happens commonly that 
it is desired to measure tissue metabolism precisely when arterial concentration is changing or when metabolism may be changing. It is therefore equally necessary to ask how much change in arterial concentration or in metabolism is tolerable if the Fick principle is to be used or whether the Fick principle can be modified to include non-steady states with respect to arterial concentration or tissue metabolism.

Many investigators have stated or implied that even though, during non-steady states, an A-V difference may not measure accurately tissue uptake or release at that time, it must be true at least qualitatively that a positive $A-V$ difference means that there is net tissue uptake, and a negative $\mathrm{A}-\mathrm{V}$ difference means that there is net tissue release. This is utterly incorrect, as we shall show.

In substance, then, we ask what interpretation can be put on $\mathrm{A}-\mathrm{V}$ differences during non-steady states.

\section{Indicator dilution theory: tissue metabolism is zero}

1. Stationary systems. The discussion which follows is based on principles developed for analysis of indicator dilution theory for measurement of blood flow and volume. Indicator dilution techniques rely on selection of a substance for which metabolism is zero; i.e., the Fick principle reduces to input $=$ output for the steady state. The importance of indicator dilution theory for the present application is that the behavior of certain transient changes in input has been analyzed.

Consider a vascular bed with a single entrance and a single exit. Between entrance and exit, branching and intercommunication of blood vessels may be as complicated as you please. The exact form of the internal structure of the system is immaterial. Blood entering the system during some time interval, say between time $t$ and $t+d t$, will not leave the system as a unit at some later time but will be dispersed with respect to time. The fraction of it which leaves the system $s$ time units later between time $t+s$ and $t+s+d s$ is $h(s) d s$, where $h(s)$ is the fraction leaving per unit time; $h(s)$ is a distribution or frequency function with dimension 1 /time. For example, if 20 per cent of blood which entered at time zero leaves the system per unit time dur- ing the fourth time interval after zero, then $h(4)$ $=0.2$. The sum of all $h(s)$ is unity; that is, sooner or later all the blood which entered at time $t$ leaves the system and $\int_{0}^{\infty} h(t) d t=1$.

The distribution function of transit times through the system is $h(s)$ or $h(t)$. If, for example, the shortest transit time is 3 time units and 30 per cent of the blood has such a transit time, 40 per cent of the blood has a transit time of 4 time units and the remaining 30 per cent of the blood has a transit time of 5 time units, then, $h(0)=0, h(1)=0, h(2)=0, h(3)=0.3$, $h(4)=0.4, h(5)=0.3, h(6)=0$. The mean transit time, $\bar{t}$, is $(3 \times 0.3)+(4 \times 0.4)+$ $(5 \times 0.3)=\int_{0}^{\infty} t h(t) d t$.

An indicator is a substance which is so dispersed through the system that the distribution function of its transit times is the same as that of the native material under investigation. That is, the distribution function of transit times of the indicator, which we can observe and measure, gives us the desired distribution function of transit times of native unmarked material which we cannot otherwise observe.

If an indicator is introduced as some function of time, $i(t)$, into the entrance of the system, its concentration at exit is described by the relation which we shall develop provided the following conditions hold: $a$ ) flow through the system is constant; $b$ ) volume of the system is constant; c) indicator is mixed thoroughly with inflowing blood at the entrance to the system so that $i(t) / F=C_{A}(t)$, that is, injected indicator divided by appropriate fluid flow is the input concentration of indicator; $d$ ) all injected indicator leaves the system eventually, that is, there are no stagnant pools; $e$ ) the system is stationary, that is, the distribution function of transit times does not change during the period of observation. (This is a more strenuous limitation than demanding constant flow and volume, because it is possible to vary the distribution function and still hold flow and volume constant.)

We wish to describe concentration of indicator at exit at time $t, C_{V}(t)$, as a function of concentration of indicator at entrance, $C_{A}(t)$. Consider the contribution to the exit concentration at time $t$ made by indicator which entered the 
system $s$ time units earlier, that is, at time $(t-s)$. The fraction of injected indicator leaving the system per unit time is $h(s)$. The amount of it leaving the system during the interval between $s$ and $s+d s$ time units after introduction is $C_{A}(t-s) \cdot h(s) \cdot d s$. Now let $s$ vary from 0 to $t$ and sum $C_{A}(t-s) \cdot h(s) \cdot d s$ over all such time intervals. The sum is the desired concentration at exit, or

$$
C_{V}(t)=\int_{0}^{t} C_{A}(t-s) h(s) d s
$$

Equation 1 is owing to Stephenson (6) and further discussion of it may be found in References 7-9. For present purposes we are interested in examining the effect on A-V differences of several functions that $C_{A}(t)$ might assume.

Consider first the behavior of Equation 1 when $C_{A}(t)$ is a constant $=I / F$ for $t \geq 0$ and $=0$ for $t<0$, where $I$ is the constant rate of injection of indicator and $F$ is constant blood flow. Equation 1 becomes

$$
C_{V}(t)=\frac{I}{F} \int_{0}^{t} h(s) d s
$$

Because $\int_{0}^{\infty} h(t) d t=1, \operatorname{limit}_{t \rightarrow \infty} C_{V}(t)=\frac{I}{F} \cdot C_{V}(t)$ approaches that limit along the curve that is proportional to the cumulative frequency function of transit times, $\int_{0}^{t} h(s) d s=H(t)$. The cumulative frequency function of transit times describes the fraction of blood which has traversed the system by time $t$. Thus, if 30 per cent of the blood has a transit time of 3 time units, 40 per cent has a transit time of 4 time units and none has any shorter transit time, then at the end of the fourth time unit 70 per cent of the blood which entered at time zero has left the system, or $H(4)=0.7$. The limit of $H(t)$, of course, is 1 .

Depending on the exact nature of $h(t), C_{V}(t)$ will look something like the curve drawn in Figure, 1 . The A-V difference $C_{A}(t)-C_{V}(t)$ $=(I / F)-C_{V}(t)$, is positive from time zero until some time at which the last particle of that indicator which was injected at zero time has left the system. At that instant $H(t)=1$.

It is obvious, despite the fact that there is a period of time during which $C_{V}(t)<C_{A}(t)$, that there has been no tissue metabolism of indicator;

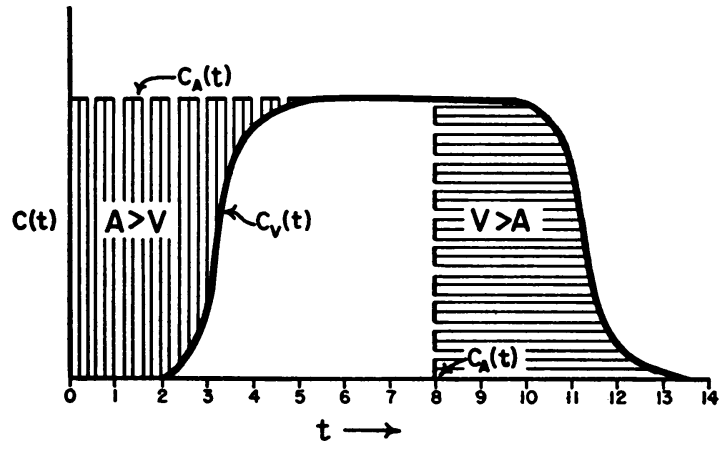

Fig. 1. Indicator dilution curve. Flow constant, and stationarity exists. Constant injection.

that is, a positive A-V difference does not necessarily mean that there has been uptake of the substance by tissues. In the case at hand, the area between the arterial and venous concentrations (see Figure 1) is exactly the mean transit time multipled by the steady state concentration of indicator, $I / F$ (see Reference 7 for proof). The mean transit time, $\bar{t}$, is exactly the volume, $V$, in which indicator is distributed, divided by the flow, $F$, through the system. The integrated $\mathrm{A}-\mathrm{V}$ difference is therefore proportional to $V / F$.

If at some time, $T$, after the steady state has been reached, injection of indicator ceases, arterial concentration at entrance falls immediately to zero. We now imagine that indicator is washed out of the system by indicator-free fluid and the equation becomes

$$
C_{V}(t)=\left\{\begin{array}{l}
0, t<0 \\
\frac{I}{F} \int_{0}^{t} h(s) d s, 0 \leq t \leq T \\
\frac{I}{F}\left(1-\int_{T}^{t-T} h(s) d s\right), t \geq T
\end{array}\right.
$$

Figure 1 demonstrates Equation 3. After time $T, C_{V}(t)$ exceeds $C_{A}(t)$ until all indicator is washed out of the system. This negative A-V difference, of course, does not mean that some tissue is producing the indicator and delivering it to the circulation. Indeed, the integrated negative $A-V$ difference is exactly the same size as the integrated positive $\mathrm{A}-\mathrm{V}$ difference and is a measure of $\bar{t}$.

There is nothing in this analysis which restricts the indicator to the vascular system. If one uses an indicator which diffuses into inter- 
stitial space, so long as the original assumptions are met, one simply changes the distribution function of transit times to include extravascular paths. The flow remains the same. The volume measured is larger than that of the vascular system because it is the volume of distribution of indicator. The integrated $\mathrm{A}-\mathrm{V}$ difference from time zero to $T$ is therefore larger, but there is still no measure of tissue metabolism. The indicator may be distributed throughout tissue water. It will still obey Equation 3. The integrated $\mathrm{A}-\mathrm{V}$ difference from time zero to $T$ will be larger. It may even exceed the $A-V$ difference of those metabolized substances which have an apparent volume of distribution less than that of total water.

Now let us consider the behavior of Equation 1 when $C_{A}(t)$ is not constant. A simple case lets $C_{A}(t)$ increase with constant velocity, that is,

$$
C_{A}(t)=\left\{\begin{array}{l}
0, t<0 \\
a \cdot t, t \geq 0
\end{array}\right.
$$

where $a$ is a constant. Equation 1 becomes

$$
\begin{aligned}
C_{V}(t)=a & \int_{0}^{t}[t-s] h(s) d s \\
& =a t \int_{0}^{t} h(s) d s-a \int_{0}^{t} s h(s) d s
\end{aligned}
$$

The asymptotic behavior of $C_{V}(t)$ is definite from Equation 4 because $\lim _{t \rightarrow \infty} \int_{0}^{t} h(s) d s=1$ and $\lim _{t \rightarrow \infty}$

$$
\begin{aligned}
& \int_{0}^{t} s h(s) d=\bar{t} . \text { Therefore, } \\
& \lim _{t \rightarrow \infty} C_{V}(t)=a \cdot(t-\bar{t})=C_{A}(t-\bar{t})
\end{aligned}
$$

that is, when all indicator introduced at time zero is washed out of the system, then $C_{V}(t)$ equals the arterial concentration introduced $\bar{t}$ time units earlier and $C_{V}(t)$ continues to be so related to $C_{A}(t)$ from then on. There will, therefore, always be a positive $A-V$ difference even though there is obviously no tissue metabolism of indicator.

Now let $C_{A}(t)$ decrease linearly from some previously constant concentration; that is,

$$
C_{A}(t)=\left\{\begin{array}{l}
C_{A}(0), t \leq 0 \\
C_{A}(0)-a \cdot t, 0 \leq t \leq C_{A}(0) / a \\
0, t \geq C_{A}(0) / a
\end{array}\right.
$$

Then Equation 1 becomes

$$
\begin{aligned}
& C_{V}(t)=C_{A}(0) \\
& \quad-a \int_{0}^{t}[t-s] h(s) d s, \quad 0 \leq t
\end{aligned}
$$

and the asymptotic behavior, until $C_{A}(t)=0$ at $t=C_{A}(0) / a$, is

$$
\lim _{t \rightarrow \infty} C_{V}(t)=C_{A}(t-\bar{t})
$$

The A-V difference, once $C_{V}(t)$ reaches the above value, is $C_{A}(t)-C_{V}(t)=-a \bar{t}$; that is, the $\mathrm{A}-\mathrm{V}$ difference is always negative.

It is instructive to combine a rising and falling arterial injection so that the envelope of arterial concentration is triangular with time, as in Figure 2. Then

$$
C_{A}(t)=\left\{\begin{array}{l}
0, \quad t \leq 0 \\
a \cdot t, \quad 0 \leq t \leq T \\
a \cdot(2 T-t), \quad T \leq t \leq 2 T \\
0, \quad t \geq 2 T
\end{array}\right.
$$

Substitution of the appropriate values of $C_{A}(t$ $-s)$ into Equation 1 yields the curve of $C_{V}(t)$ for $a=1$ and for the function $h(t)$ illustrated in Figure 2. The area under the curve $C_{A}(t)$ equals the area under the curve $C_{V}(t)$ because all injected indicator must leave the system. $F \int_{0}^{\infty} C_{A}(t) d t$ is the total indicator injected and $F \int_{0}^{\infty} C_{V}(t) d t$ is the total indicator leaving the system. It follows immediately, therefore, that the shaded area marked $A>V$ in Figure 2 equals the shaded area marked $V>A$. The points to

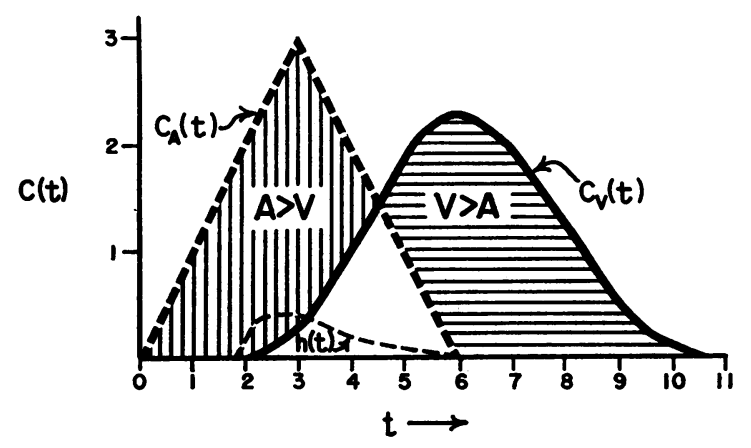

Fig. 2. Indicator DILUTion CURVE. Flow constant, and stationarity exists. $C_{A}(t)$ increases linearly, then decreases linearly. 
be noted in Figure 2 are: that the maximal venous concentration is less than the maximal arterial; that until 4.5 time units $C_{A}>C_{V}$, although there is no tissue uptake, and after 4.5 time units $C_{V}>C_{A}$, although there is no tissue production; and that $C_{V}$ continues to rise for a time after $C_{A}$ has begun to fall.

We can now make a general statement about $\mathrm{A}-\mathrm{V}$ differences of indicators during transient changes in indicator input effected by altered $C_{A}(t)$. Assume that $C_{A}(t)$ and $C_{V}(t)$ are constant for a sufficiently long time before $t=0$ and that at $t=0$ a change in $C_{A}(t)$ begins. Equation 1 can be integrated by parts to yield

$$
\begin{aligned}
C_{V}(t)= & \int_{0}^{t} C_{.1}(t-s) h(s) d s \\
= & \left.C_{A}(t-s) H(s)\right|_{0} ^{t} \\
& \quad+\int_{0}^{t} C^{\prime}{ }_{A}(t-s) H(s) d s
\end{aligned}
$$

where $H(t)$ is the cumulative distribution function $\int_{0}^{t} h(s) d s, C^{\prime}{ }_{A}$ is the first derivative of $C_{A}$ and $d C_{A}(t) / d t=-d C_{A}(t-s) / d s$.

Because $H(0)=0$ in the real vascular system, $\left.C_{A}(t-s) \quad H(s)\right|_{0} ^{t}=C_{A}(0) H(t)$.

Because $H(t) \leq 1$, if $C^{\prime}{ }_{A}(t)>0$ (that is, if $C_{A}(t)$ is increasing with time), $\int_{c}^{t} C^{\prime}{ }_{A}(t-s)$ $H(s) d s<\int_{0}^{t} C^{\prime}{ }_{A}(t-s) d s=C_{A}(t)-C_{A}(0)$.

Substituting these functions in the integration by parts yields $C_{V}(t)<C_{A}(0) H(t)+C_{A}(t)$ $-C_{A}(0)=C_{A}(t)-C_{A}(0)[1-H(t)]$, or $C_{V}(t)$ $<C_{A}(t)$.

If arterial concentration is decreasing with time, $\quad C^{\prime}{ }_{A}(t)<0$ and $\int_{0}^{t} C^{\prime}{ }_{A}(t-s) H(s) d s$ $>\int_{0}^{t} C^{\prime}{ }_{A}(t-s) d s$. With this substitution and by argument similar to that used above, $C_{V}(t)>C_{A}(t)$.

Thus, if arterial concentration increases there is a positive $\mathrm{A}-\mathrm{V}$ difference. If arterial concentration decreases there is a negative $A-V$ difference. If arterial concentration is constant for a sufficiently long period (that is, long enough for $H(t)$ to become unity) then venous concen- tration will, when $H(t)=1$, equal arterial concentration. A-V differences occur because positive times are required for traversal of the vascular net (that is, because $H(0) \neq 1$ ). Clearly, then, $\mathrm{A}-\mathrm{V}$ differences during non-steady states are not in themselves evidence of tissue metabolism.

2. Nonstationary systems; variable flow. We have so far been concerned with input changes produced by altered concentration at constant flow. Now consider input changes produced by altered flow.

Consider first the simplest but least likely case, in which flow is altered without altering the distribution of transit times (that is, stationarity exists). This is possible if the relation $V / F=\bar{t}$ through every path is constant. Such an event is handled simply by generalizing Equation 1

$$
m_{0}(t)=\int_{0}^{t} m_{i}(t-s) h(s) d s
$$

where $m_{0}(t)$ is output (= flow at time $t$ multiplied by venous concentration at time $t$ ) at time $t$ and $m_{i}(t)$ is input at time $t$. If $F_{0}(t)$ is flow at output, $F_{i}(t)$ is inflow, and $C_{A}$ is the constant arterial concentration, then Equation 7 becomes

$$
C_{V}(t)=\frac{C_{A}}{F_{0}(t)} \int_{0}^{t} F_{i}(t-s) h(s) d s .
$$

Since nothing has been done to alter concentration anywhere in the system, it must be that $C_{V}(t)=C_{A}$. Therefore,

$$
F_{0}(t)=\int_{0}^{t} F_{i}(t-s) h(s) d s
$$

In a system of rigid tubes containing incompressible fluid such as water, flow everywhere in the system is identical at any time. In particular, $F_{0}(t)=F_{i}(t)$, the limiting case in which $h(t)$ is simply a brief spike beginning at $t=0$.

More realistically we must consider that a change in flow is associated with a change in the distribution of transit times through the system, that is, there is nonstationarity. Then if flow, $F$, is a function of time, $F(t)$, so is the distribution of transit times a function, not only of elapsed time following introduction of indicator, but also of absolute time at which indicator was introduced. The problem has been explored by Sherman (10) following methods used by Zadeh 
(11) in analysis of variable or dynamic electrical networks which depend on specified combinations of input, output and time. The following development relies largely on the work of Zadeh and of Sherman.

We have until now defined the time, or the center of gravity of the time interval at which indicator is added to the system, as zero time. Let us now permit this time of indicator addition to be any time, $x$. Then $h(x)$ is the distribution function of transit times through the system which exists at time $x$. But the distribution function of transit times is also a function of the time elapsed since addition of indicator, a time which we will designate $a$, where $a=t-x$, the difference between absolute time, $t$, and time of indicator addition. The distribution function is therefore $h(x, a)=h(x, t-x)=h(t-a, a)$.

Because $h(x, a)$ is a distribution function (that is, because all indicator introduced at any given time $x$ must eventually leave the system) $\int_{x}^{\infty} h(x, t-x) d t=\int_{x}^{\infty} h(t-a, a) \quad d t=1$

Now consider that $h(x, a)$ is the distribution function not only of indicator particles but of all fluid that entered the system at any given time $x$.

Consider the contribution to outflow at time $t$ made by fluid that entered the system at a particular time before $t, x_{1}$. Of the total inflow $F_{i}\left(x_{1}\right)$, the fraction leaving at $a$ time units later [that is, between times $t$ and $t+d t\left(=x_{1}+a\right.$ and $\left.\left.x_{1}+a+d a\right)\right]$ is $F_{i}\left(x_{1}\right) h\left(x_{1}, a\right) d a$, or $d F_{0}(t)=F_{i}\left(x_{1}\right) h\left(x_{1}, a\right) d a$. The total outflow is obtained simply by integrating the above expression for $d F_{0}(t)$, letting $x$ vary over all previous times before $t$, or

$$
F_{0}(t)=\int_{-\infty}^{t} F_{i}(x) h(x, t-x) d x
$$

It is implicit in Equation 9 that once a particle enters the system at any given time $x$ it is committed to traverse the system at some given transit time $a$ with the probability defined by $h(x, a)$, and that changes in flow occurring at some other $x$, and therefore changes in $h(x, a)$ do not affect transit times of particles already in the system. Further, as Sherman pointed out $(10), F_{0}(t)$ is defined only in terms of $F_{i}(x)$ and not in terms of $F_{i}(t)$, the simultaneous inflow.

By an argument analogous to that used to develop Equation 9, output of indicator as a function of input and time is

$$
\begin{aligned}
F_{0}(t) C_{V}(t) & \\
= & \int_{-\infty}^{t} F_{i}(x) C_{A}(x) h(x, t-x) d x
\end{aligned}
$$

For the case of constant arterial concentration $C_{A}$, venous concentration at time $t$ is

$$
C_{V}(t)=\frac{C_{A}}{F_{0}(t)} \int_{-\infty}^{t} F_{i}(x) h(x, t-x) d x=C_{A}
$$

which is expected because $C_{A}$ is neither diluted nor concentrated as it passes through the system.

For the special case in which $F_{i}(x)$ and $C_{A}(x)$ vary inversely so that their product is constant, $I$, Equation 10 becomes

$$
C_{V}(t)=\frac{I}{F_{0}(t)} \int_{-\infty}^{t} h(x, t-x) d x
$$

After sufficiently long time, because the integral in Equation 11 becomes unity, limit of $C_{V}(t)$ $=I / F_{0}(t)$. Until that time is reached, there will be an $\mathrm{A}-\mathrm{V}$ concentration difference.

We can define precisely what is meant by a sufficiently long time with the aid of Figure 3. Several possible distribution functions are illustrated as histograms by $\mathrm{A}, \mathrm{C}$, and $\mathrm{F}$. The total area of each distribution function is unity. Experimentally, these distribution functions are determined by injecting quickly a single quantity of indicator into the entrance to the system under study and measuring its concentration at exit during a period of stationarity. In communication engineering this is equivalent to applying a brief pulse to the input to a network and observing the response at output. In B the unit pulse is applied repeatedly, as indicated by the arrows, or in a hydrodynamic system indicator is injected at constant rate. Output distribution functions are superposed until the height of the output response becomes unity, and it remains unity as long as constant input is maintained. Although when output response is unity it equals the unit input, the output response does not represent any single unit input admitted at some earlier time but it is made up of contributions from a number of earlier inputs. The time at which the output response reaches unity is exactly the longest transit time in the system, 

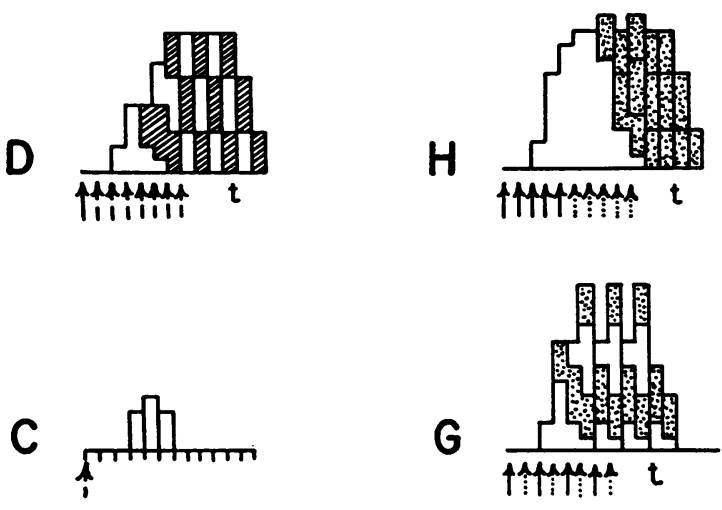

G

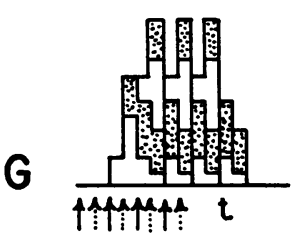

B

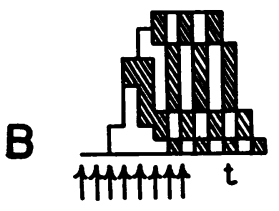

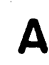

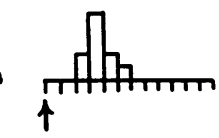

$\boldsymbol{F}$

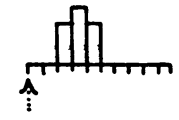

E

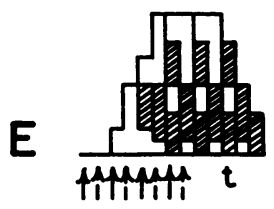

Fig. 3. VARIOUS DISTRIBUTION FUNCTIONS AND THEIR COMBINATIONS IN STATIONARY AND NONSTATIONARY STATES. Alternating white and shaded areas represent distribution of materials entering at successive time intervals. Each arrow style represents a different distribution function pertinent to entrance time indicated by arrow. The functions are: A. $h(x, a)=h_{1}(t) . \quad$ B. $\int_{0}^{\cdot t} h(s) d s=\int_{x_{1}}^{\bullet t} h(x, t$ $-x) d x$, where $h(s)=h_{1}(t) . \quad$ C. $h(x, a)=h_{2}(t) . \quad$ D. $\int_{x_{1}}^{t} h(x, t-x) d x$, where $h\left(x_{1}\right)=h_{1}(t), h\left(x_{2}\right)$ to $h\left(x_{8}\right)$ $=h_{2}(t)$. E. $\int_{x_{1}}^{t} h(x, t-x) d x$, where $h\left(x_{1}\right)=h\left(x_{3}\right)$ $=h\left(x_{5}\right)=h\left(x_{7}\right)=h_{1}(t)$ and $h\left(x_{2}\right)=h\left(x_{4}\right)=h\left(x_{6}\right)$ $=h\left(x_{8}\right)=h_{2}(t)$. F. $h(x, a)=h_{3}(t)$. G. $\int_{x_{1}}^{t} h(x, t-x) d x$, where $h_{1}(t)$ and $h_{3}(t)$ exist at alternate time units. H. $\int_{x_{1}}^{t} h(x, t-x) d x$, where $h\left(x_{1}\right)$ to $h\left(x_{5}\right)=h_{1}(t), h\left(x_{6}\right)$ to $h\left(x_{10}\right)=h_{3}(t)$.

determined from the single sudden injection curve A.

In Figure 3D, constant injection of indicator occurs but the system is nonstationary in that the distribution function changes from $h_{1}(t)$ during the first time interval to $h_{2}(t)$ for all subsequent time intervals. Output, which is superposed distribution functions, reaches unity at a time $t$ exactly equal to $x_{2}$, the time at which the distribution function through the system changed from $h_{1}$ to $h_{2}$, plus the longest transit time in the function $h_{2}(t)$ or in $h_{1}(t)$, whichever is longer.

In Figure $3 \mathrm{E}$ and $\mathrm{G}$ there is constant injection through a nonstationary system. In both cases the distribution function alternates between two functions. In $\mathrm{E}$, the longest transit time of both functions is the same. The output reaches unity exactly at that longest transit time and remains there. In $G$, the longest transit time of one distribution function differs from that of the other. The output response eventually (at a time equal to the greater of the longest transit times of the two distribution functions) oscillates symmetrically about unity, exceeding unity by as much as it is alternately less than unity. The frequency of output oscillation is exactly that at which the two distribution functions alternate.

In Figure $3 \mathrm{H}$, the system changes from one steady state, in which the distribution function is $h_{1}(t)$ to a second state in which the distribution function is $h_{2}(t)$. The time required for output to restabilize at unity after the change is made is exactly the longest transit time in the new distribution function.

To return to Equation 11, the sufficiently long time required for $C_{V}(t)$ to equal $I / F_{0}(t)$ is either the longest transit time of any distribution function which describes the system for a time at least equal to its longest time or, if the system is always nonstationary but oscillates in some regular way about a group of distribution functions, it is the longest of all transit times described by any distribution function and $C_{V}(t)$ can be taken as a weighted mean of its oscillating value.

The relation between flow and the distribution function may be confusing. It is possible, but not likely, that a change in the distribution of transit times could occur without a change in flow and it has already been stated that a change in flow could occur without a change in distribution function if volume changed appropriately, but this is again unlikely. Therefore, unless it can be demonstrated otherwise in a specific case, whenever flow changes it is safer to assume that the distribution function also changes and Equation 10 is more apt to be descriptive of the real system than is Equation 7.

But the basic assumption underlying Equa- 
tion 10 is probably an oversimplification so that even Equation 10 may be inadequate. The assumption that distribution functions are superposable rests formally on the assumption that the system is linear; that is, that the relation between input and output can be described by a linear equation. This assumption implies that once a particle has entered the system it is committed to traverse it in a given transit time; that is, changes in the distribution function of transit times are assumed not to affect particles already in the system but only new particles just entering the system.

This assumption is not apt to be valid in general. When the distribution function changes owing to vasoactivity, for example, the transit times of particles already in the system are probably changed. In the extreme, with closure of some portions of a capillary bed, flow ceases and diffusion, which until now has been considered negligible, becomes the only means by which solute particles can move through the system; that is, movement of solute and solvent, previously considered to be coupled and described by a common distribution function, becomes uncoupled so that one distribution function does not describe transit times through the system. The system is, therefore, probably formally nonlinear and the development of Equation 10, which rests on the superposition principle, is inappropriate. There is no simple formal solution to this problem. A description of the relations between input, output and time in such a nonlinear system requires detailed knowledge of the behavior of every particle within the system as a function of position within the system and time, and there is no experimental method for obtaining the necessary information at present. This means that in nonstationary and nonlinear systems there is no way to relate simultaneous arterial and venous concentrations, and no simple correction factor, such as evolved from discussion of Equation 4, is applicable.

\section{Tissue metabolism not zero}

1. Tissue uptake. We have been examining in increasing complexity A-V concentrations of substances for which tissue uptake or output is zero. The problem is even more difficult when we let tissue uptake assume nonzero values.
In the first place, the distribution of transit times of a substance, some of which is metabolized, is different from the distribution of transit times of a solute confined to plasma or to total extracellular fluid and is even apt to be different from that of total water. The transit times under consideration are only of those particles which leave the system and so contribute to the venous concentration. If tissue uptake from every channel through the system were proportional to the rate at which the metabolizable substance flowed through that channel, then and only then, would the distribution of transit times of the substance be identical with the distribution of transit times of some nonmetabolized indicator. Although there may be in certain special cases a close relation between vascularity and metabolism, in general, we anticipate that the fractional uptake from some channels will be different from that in others. There might be little uptake from blood through channels with very short transit times, the extreme being the case of arteriovenous shunts, and there might be a great deal of uptake from blood coursing through intricate capillary nets with long transit times.

For example, if $h(t)$, the distribution function of indicator transit times is $h(0)=0, h(1)=0$, $h(2)=0.3, h(3)=0.4, h(4)=0.3, h(5)$, and so forth $=0$, then the distribution function of transit times, $g(t)$, of a metabolized substance through the same vascular bed might be $g(0)$ $=0, \quad g(1)=0, \quad g(2)=0.5, \quad g(3)=0.3, \quad g(4)$ $=0.2, g(5)$, and so forth $=0$. In this example, the mean transit time for nonmetabolized particles, $\int_{0}^{\infty} t h(t) d t$, is longer than the mean transit time for metabolized particles, $\int_{0}^{\infty} t g(t) d t$, because a large fraction of metabolized particles coursing through long-transit-time channels was eliminated by metabolism within the system.

The purpose of this example is to make it clear that no simple known relation exists between transit times of indicators and of metabolities. It is therefore not possible to "correct" observed venous concentrations by displacement along the time axis in accordance with some notion of the distribution of transit times of nonmetabo- 
lized indicators, although the correction can be made as a reasonable approximation in those cases in which nearly all of the substance traverses the system; that is, in those cases in which only a small fraction of entering material is taken up by tissues. For example, in the forearm of man at rest in the basal state about 98 per cent of glucose delivered by arterial blood appears in forearm venous effluent (12). In this example, then, $g(t)$ is probably very nearly the same as $h(t)$ so that for certain practical purposes it would be acceptable to assume that $g(t)$ was indeed $h(t)$.

The relation between $h(t)$ and $g(t)$ is easily defined. Consider those particles whose transit times are between $t$ and $t+d t$ time units. Of those nonmetabolizable or indicator particles entering at zero time, the fraction leaving in the time interval $t$ to $t+d t$ is $h(t) d t$. Of metabolizable particles, the fraction of those that entered at zero time which leave in the same time interval is $r(t) h(t) d t$, where $0 \leq r(t) \leq 1$. Integration over all time yields the fraction of metabolizable particles which entered at zero time and which eventually leave the system, $p=\int_{0}^{\infty} r(t) h(t) d t$ and $0 \leq p \leq 1$. Therefore, of those metabolizable particles entering at zero time and which are not metabolized, the fraction leaving the system per unit time between $t$ and $t+d t$ time units later is

$$
g(t)=r(t) h(t) / p
$$

When $g(t)=h(t)$, from Equation $12 r(t)=p$, a constant, or $g(t)$ can equal $h(t)$ only if $r(t)$ is constant. When $p$ is almost unity, there can be no assurance that any given $g(t) \doteq h(t)$, but $\int_{0}^{t} C_{A}(t-s) g(s) d s \doteq \int_{0}^{t} C_{A}(t-s) h(s) d s$ for sufficiently large $t$.

One can, during steady states, observe the distribution function of transit times of certain metabolizable substances by using tracer quantities of labeled material otherwise identical with the metabolized substance. This distribution function, $g(t)$, can be evaluated only while there is stationarity. This requires not only that flow and volume remain constant but also that there be constant uptake of metabolite per unit time, $\dot{Q}$, for if rate of uptake changes with time it cannot be assumed that the proportional change in every channel is identical, which is required if $g(t)$ is to be the same.

The quantity of metabolite entering the system between time $t$ and $t+d t$ is $F C_{A}(t) d t$. Of this quantity, assuming uptake is constant, the total amount leaving the system during all subsequent time intervals is $\left[F C_{A}(t)-\dot{Q}\right] d t$. By the arguments used to develop Equation 1

$$
\begin{aligned}
C_{V}(t) & =\int_{0}^{t}\left[C_{A}(t-s)-\frac{\dot{Q}}{F}\right] g(s) d s \\
& =\int_{0}^{t} C_{A}(t-s) g(s) d s-\frac{\dot{Q}}{F} G(t)
\end{aligned}
$$

where $G(t)=\int_{0}^{t} g(s) d s$, and $F C_{A}(t) \geq \dot{Q}$.

In a form analogous to that of the classical Fick equation, Equation 13 becomes

$$
\dot{Q}=\frac{F}{G(t)}\left[\int_{0}^{t} C_{A}(t-s) g(s) d s-C_{V}(t)\right]
$$

In the discussion of Equation 6 we showed that if $d C_{A}(t) / d t>0, \int_{0}^{t} C_{A}(t-s) g(s) d s<C_{A}(t)$, and if $d C_{A}(t) / d t<0, \int_{0}^{t} C_{A}(t-s) g(s) d s$ $>C_{A}(t)$. Therefore, after sufficient time has elapsed so that $G(t)$ has passed to unity, if $C_{A}(t)$ is increasing, the A-V difference overestimates $\dot{Q}$, and if $C_{A}(t)$ is decreasing the A-V difference underestimates $\dot{Q}$. Indeed it is even possible for the A-V difference to be negative, if $d C_{A}(t) / d t$ is sufficiently negative and the mean transit time is sufficiently long, despite the fact that there may be real tissue uptake of the substance measured.

Except where $g(t)=h(t)$, it is not possible to use an indicator curve, such as that in Figure 2, as a guide in estimation of $\dot{Q}$ during transient changes in $C_{A}(t)$. For example, if in Figure 2 $C_{A}(t)$ were displaced upward, as in Figure 4, by some constant for all $t$ so that there was a constant steady state A-V difference, the A-V difference during the transient would not be that of Equation 12 unless the distribution function, $h(t)$, of the indicator happened to be the same as that of the metabolite, $g(t)$. If $g(t)$ can be determined independently and can be proven 


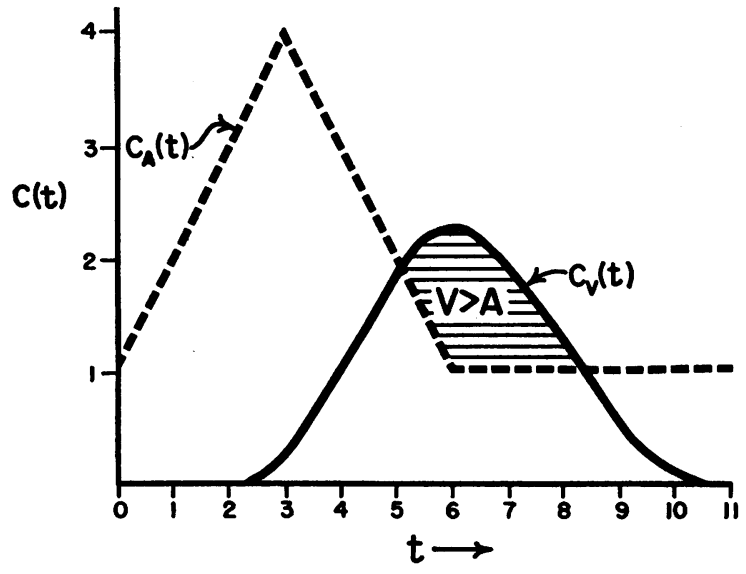

Fig. 4. Constant uptake of a substance with SAME CHANGES IN ARTERIAL CONCENTRATION AS IN FIGURE 2. Uptake cannot be calculated accurately at any time during changes in arterial or venous concentration. Flow multiplied by integrated $\mathrm{A}-\mathrm{V}$ difference from time 0 to 11 is, in this example, total tissue uptake over that time interval.

stationary, then Equation 13 can be used to test whether or not $\dot{Q}$ is in fact constant.

If tissue uptake is not constant but is some function of time, $\dot{Q}(t)$, then we must consider that the system is not stationary. Consider first the more general problem of varying flow and varying uptake.

If we assume that the system is linear-that is, that the distributions of inputs are superposableas we did in development of Equation 10 when we considered effects of altered flow and distribution function on indicator concentration, we can immediately generalize Equation 10 by analogy

$$
m_{0}(t)=\int_{-\infty}^{t} p(x) m_{i}(x) g(x, t-x) d x
$$

where $m_{0}(t)=F_{0}(t) C_{V}(t), m_{i}(t)=F_{i}(t) C_{A}(t)$ and $p(t)$ is that fraction of $m_{i}(t)$ which is not metabolized (see Equation 12). For constant uptake, Equation 14 becomes

$$
m_{0}(t)=\int_{-\infty}^{t}\left[m_{i}(x)-\dot{Q}\right] g(x, t-x) d x
$$

and for constant arterial concentration, Equation 15 becomes

$$
\begin{aligned}
C_{V}(t)=\frac{C_{A}}{F_{0}(t)} & \int_{-\infty}^{t} F_{i}(x) g(x, t-x) d x \\
& -\frac{\dot{Q}}{F_{0}(t)} \int_{-\infty}^{t} g(x, t-x) d x
\end{aligned}
$$

Unlike the case of indicator dilution at constant arterial concentration and varying flow, in which $C_{A}(t)=C_{A}$, a constant (see discussion following Equation 10), when there is tissue uptake a change in flow produces a change in venous concentration even if arterial concentration is held constant. It is intuitively evident that this must also be true in nonlinear systems. Figure 5 illustrates the sort of changes one might find when flow is changed in stepwise fashion so that the system moves from one steady state to another. An increase in inflow, $C_{A}$ and $\dot{Q}$ remaining constant, produces a transient change in outflow and in venous concentration, which lag behind input changes. Owing to the lag there is a transient increase in A-V difference and $\dot{Q}$ is overestimated. The steady state is reached at a time equal to the longest transit time of metabolizable particles through the system, not the longest transit time of plasma. The overestimate in $\dot{Q}$ is not compensated in any way by a subsequent reduction in $\mathrm{A}-\mathrm{V}$ difference as long
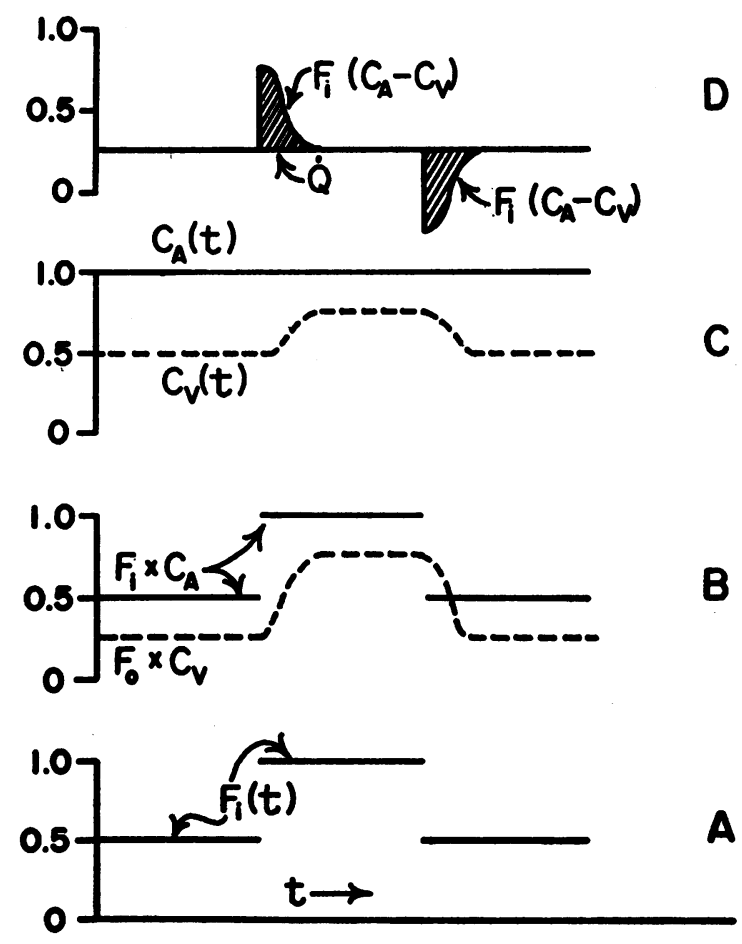

Fig. 5. EFFEct OF ChANGE IN FLOW ON A-V DifferENCE. A. Step increase in flow. B. Step increase in input with consequent change in output. C. Change in output concentration with change in flow, despite constant arterial concentration and constant uptake. D. Although tissue uptake, $\dot{Q}$, is constant, the product of flow and $\mathrm{A}-\mathrm{V}$ difference is not constant when there is a step change in flow. 
as the new steady state is maintained. This does not mean that there is tissue storage of the apparent excess, but that there is always a lag between input and output so that $F_{0}(t) C_{V}(t)$ is not made up of those particles that entered at $t$, but of those that entered over some time period before $t$. When flow is decreased to the original rate, there is a transient decrease in $\mathrm{A}-\mathrm{V}$ difference and $\dot{Q}$ is underestimated. Indeed, if the decrease in flow is sufficiently great there may even be negative $\mathrm{A}-\mathrm{V}$ differences, as in the illustration, and it may be concluded erroneously that there is tissue output. If, when flow returns to the original rate, the distribution of transit times also returns to the original distribution, the overestimate of $\dot{Q}$ which occurred when flow increased is exactly the same as the underestimate of $\dot{Q}$ which occurred when flow decreased. The integrated $\mathrm{A}-\mathrm{V}$ difference multiplied by flow during the entire time interval will therefore measure total uptake over that time interval.

Although the assumption of linearity is not apt to hold when the system is nonstationary owing to altered flow, for reasons discussed earlier, the system may very well remain linear when nonstationarity is owing to altered metabolism in the presence of constant flow. This greatly simplifies the problem, as follows.

For the case of constant flow Equation 14 becomes

$$
C_{V}(t)=\int_{-\infty}^{t} p(x) C_{A}(x) g(x, t-x) d x
$$

Equation 17 does not tell us what tissue uptake was at time $t$. Although the quantity of material entering between time $t$ and $t+d t$ which is metabolized eventually is $(1-p) F C_{A}(t) d t$, this quantity is not $\dot{Q} d t$ because there is a distribution of transit times from entrance to the various sites of metabolism within the system. By analogy with equations developed previously in this paper for nonstationary linear systems, rate of tissue uptake as a function of time

$$
\begin{aligned}
\dot{Q}(t)= & F \int_{-\infty}^{t}[1-p(x)] C_{A}(x) m(x, t-x) d x \\
= & F \int_{-\infty}^{t} C_{A}(x) m(x, t-x) d x \\
& -F \int_{-\infty}^{t} p(x) C_{A}(x) m(x, t-x) d x
\end{aligned}
$$

where $m(x, a)$ is the distribution function of transit times from entrance to sites of uptake.

The integral in the second term on the righthand side of Equation 18 is obviously related to the integral of Equation 17 which describes $C_{V}(t)$, the difference being in the distribution functions. The integral in the first term of Equation 18 is clearly related to $C_{A}(t)$, but it is also clearly not $C_{A}(t)$. Equation 18 , in short, is a general form of the classical Fick equation for constant flow but for variable arterial concentration and variable uptake.

When $C_{A}(t)$ is constant, $C_{A}$, Equation 18 becomes

$$
\begin{aligned}
\dot{Q}(t)=F & C_{A} \int_{-\infty}^{t} m(x, t-x) d x \\
& -F C_{A} \int_{-\infty}^{t} p(x) m(x, t-x) d x
\end{aligned}
$$

After a sufficiently long time, the first term in Equation 19 becomes $F C_{A}$. If $p(t)$ is also constant Equation 19 becomes $\dot{Q}(t)=F C_{A}(1-p)$ $\int_{-\infty}^{t} m(x, t-x) d x$, and for $t$ sufficiently large, limit $\dot{Q}(t)=F C_{A}(1-p)=F\left(C_{A}-C_{V}\right)$, which is the classical Fick equation.

Equation 18 is unfortunately not very useful in that form because it contains the distribution function, $m(x, a)$, which is not subject to direct experimental investigation. An approximate evaluation of $m(x, a)$ comes from the following analysis.

Consider what would have been the distribution of transit times, $f(t)$, through the system of only those particles which were removed. That is, if their transit times had not terminated within the system as described by $m(t)$, what would have been their transit times? Refer to the argument by which Equation 12, relating $g(t)$ to $h(t)$, was developed. Clearly,

$$
f(t)=\frac{[1-r(t)] h(t)}{1-p}=\frac{h(t)-p g(t)}{1-p}
$$

Some relation must exist between the transit time of a particle to the point of uptake and its transit time through the entire system. It is plausible to assume that points of uptake lie approximately midway in time between entrance and exit. On this assumption, $m(2 t)=f(t)$ 
and Equation 18 becomes

$$
\begin{aligned}
\dot{Q}(t) & \doteq F \int_{-\infty}^{t} C_{A}(x) h\left(\frac{t-x}{2}\right) d x \\
& -F \int_{-\infty}^{t} C_{A}(x) p(x) g\left(x, \frac{t-x}{2}\right) d x
\end{aligned}
$$

Equation 20 is much simpler to handle than Equation 18. The first term on the right-hand side deals only with a stationary system, that is, as long as flow is constant (and volume), the distribution of transit times of the appropriate indicator, described by $h(t)$, is not dependent on time. We have already seen how to handle such a function when we considered indicator dilution through stationary systems. The second integral on the right-hand side of Equation 20 is closely related to $C_{V}(t)$, defined in Equation 17 . Indeed, if the system becomes stationary for a sufficiently long time (the longest transit time) the second term on the right-hand side of Equation 20 equals $F C_{V}(t)$.

From the discussion surrounding Figure 2 and Equations 4-6, after sufficiently long time, for the case of linear increase in $C_{A}(t)$, which is a useful case because many changes in arterial concentration may be considered linear over some practicable time interval,

$$
\dot{Q}(t) \doteq F C_{A}\left(t-\frac{1}{2} \bar{t}\right)-F C_{V}\left(t+\frac{1}{2} \dot{t}_{g}\right)
$$

where $\bar{t}$ is mean time through $h(t)$ and $\bar{t}_{g}$ is mean time through $g(t)$. For the special case of $\dot{Q}(t)$ $=0, \bar{t}=\bar{t}_{g}$ and Equation 21 reduces to the limiting indicator dilution equation, $C_{V}(t)=C_{A}(t$ $-\bar{t}$.

In short, if $C_{A}(t)$ increases linearly an approximate measure of $\dot{Q}(t)$ can be obtained by moving back in time $\frac{1}{2} \bar{t}$ units and noting $C_{A}$, by moving up in time $\frac{1}{2} \bar{t}_{g}$ units and noting $C_{V}$. The difference between these two corrected concentrations is nearly equal to $\dot{Q}(t) / F ; \bar{t}_{g}$ can be obtained by tracer techniques or, for the case of substances such as glucose traversing the forearm of man, in which about 98 per cent of entering material leaves the system, $\bar{t}_{\theta}$ may be represented satisfactorily by $\bar{t}$.

From the discussion surrounding Figure 1 and Equations 2 and 3 for the case of constant arterial concentration and relatively linear changes in uptake, after sufficiently long time

$$
\dot{Q}(t) \doteq F C_{A}-F C_{V}\left(t+\frac{1}{2} \bar{t}_{g}\right)
$$

For the special case of $\dot{Q}(t)=0, \frac{1}{2} \bar{t}_{g}$ degenerates to zero because it appears in Equation 22 only as an approximation of $\dot{t}_{m}$ which, of course, is nonexistent if there is no tissue uptake. Equation 22 states that even if input to the system (flow and arterial concentration) is constant, $\dot{Q}(t)$ is not equal to $F C_{A}-F C_{V}(t)$, but venous concentration must be corrected backward in time by an amount assumed to represent mean time from entrance to points of uptake.

2. Tissue release. We have so far spoken only of uptake and not of output of metabolites. In a steady state, the excess of the constant venous concentration above constant arterial concentration represents the ratio of constant tissue output to constant flow. In non-steady states there is no such symmetry between the analysis of uptake and of output. Consider the case in which a substance is introduced into our test system by way of arterial inflow and all of it eventually leaves the system; that is, there is no uptake. The test tissue, however, releases additional quantities of the substance. The concentration at output is therefore

$$
\begin{aligned}
C_{V}(t)=\int_{0}^{t} C_{A}(t & -s) h(s) d s \\
& +\frac{1}{F} \int_{0}^{t} \dot{q}(t-s) n(s) d s
\end{aligned}
$$

where $\dot{q}(t)$ is rate of tissue release at time $t$, and $n(t)$ is the distribution function of transit times from points of release from tissue cells to the exit from the system.

If a change in $\dot{q}$ is also associated with a change in $n(t)$, that is, if the distribution of released material is not stationary, and we must assume that this is apt to be the case, it is quite likely that as long as $h(t)$ describes a stationary system, the distribution of released material can be described by a linear net. This means simply that although the sites of and relative contributions to tissue release may vary with time,once the metabolite is released it is distributed through elements of the system described by $h(t)$, which is a stationary system. By analogy with previous discussions of linear nonstationary systems, Equation 23 is immediately generalized to

$$
\begin{aligned}
C_{V}(t)=\int_{0}^{t} C_{A}(t-s) h(s) d s \\
\quad+\frac{1}{F} \int_{-\infty}^{t} \dot{q}(x) n(x, t-x) d x
\end{aligned}
$$


The first term on the right-hand side of Equation 24 is simply an indicator dilution term and obviously Equation 24 reduces to the indicator dilution Equation 1 for $\dot{q}(t)=0$. This means that all of the discussion of effect of varying arterial concentration on indicator dilution curves is applicable to Equation 24. The second term is not the rate at which tissues release the substance at time $t$, but simply the sum of all contributions made by tissue release before time $t$ which reach the sampling site at exit at time $t$.

For the case of $C_{A}(t)$ constant, Equation 24 becomes

$F\left[C_{V}(t)-C_{A}\right]=\int_{-\infty}^{t} \dot{q}(x) n(x, t-x) d x$

that is, flow multiplied by simultaneous $\mathrm{A}-\mathrm{V}$ difference is not tissue release at time $t$ but is the sum of those fractions of tissue release at time $x$ whose transit times are $(t-x)$.

If $\dot{q}(t)$ increases linearly, assuming that the mean transit time from sites of release to exit is half the mean transit time from entrance to exit, and if $C_{A}(t)$ is constant, $F\left[C_{V}(t)-C_{A}\right] \doteq \dot{q}(t$ $-\frac{1}{2}(\bar{t})$, that is, use of simultaneous $\mathrm{A}-\mathrm{V}$ difference determines the rate of tissue release which occurred approximately $\frac{1}{2} \dot{t}$ time units earlier.

If $\dot{q}(t)$ is constant but $C_{A}(t)$ is variable, $F C_{V}(t)$

$-F \int_{0}^{t} C_{A}(t-s) h(s) d s=\dot{q}$, which can be handled according to the discussion of indicator dilution curves and which reduces to the classical Fick equation for $C_{A}(t)$ constant.

If flow is not constant, the system probably becomes nonlinear and the difficulties are those encountered in the previous discussion of nonlinear systems.

3. Simultaneous uptake and release, and other complications. When tissue uptake and tissue release occur simultaneously, combination of Equations 17 and 24 yields, for constant flow,

$$
\begin{aligned}
& C_{V}(t)=\int_{-\infty}^{t} p(x) C_{A}(x) g(x, t-x) d x \\
&+\frac{1}{F} \int_{-\infty}^{t} \dot{q}(x) n(x, t-x) d x
\end{aligned}
$$

Clearly, in this case the A-V difference gives little information about metabolic events at time $t$. Even in the steady state this is a difficult problem requiring more experimental data than are needed if only uptake or only release occurs. The problem falls into a somewhat different category than those distributive problems considered in the general development of equations here. In general, if the system is homogeneous (that is, if the concentration of the metabolite under study is the same in all venous blood draining the system), then in the steady state, $F\left(C_{A}-C_{V}\right)$ is the difference between uptake and release, uptake is measured by proper use of tracer quantities of labeled metabolite, and release is obtained by difference. If the system is not homogeneous, as in the forearm of man in which concentration of certain metabolites may vary widely from vein to vein, then this simple use of A-V differences of naturally occurring and isotopic metabolite is inadequate. The solution, if one is possible, requires some knowledge of the anatomy of the system and in general involves simultaneous $\mathrm{A}-\mathrm{V}$ differences from more than one vein, and may also require simultaneous measurement of distribution of blood flow to various parts of the system and the use of $A-V$ differences of more than one molecular species. More detailed exposition of this problem is reserved for another communication.

We have, however, raised the problem of tissue heterogeneity. Clearly it is worthless, in a system with many venous effluents, all with differing concentrations, to let all calculation of metabolism depend on measurement of concentration in blood from only one vein unless it is known that nearly all the blood leaving the system is drained by the vein sampled. In many experiments it is not possible to guarantee, as a result of anatomical good fortune or surgical intervention, that all venous blood flows through the vein sampled. It is therefore necessary to sample from a sufficient number of veins to be sure that most of the venous flow has been represented by the sampling, and it is necessary to know the contribution to total outflow made by blood flow through each bed represented by each vein sampled.

For the case of multiple exits from the system and heterogeneity of tissue metabolism, the flow which must be determined is not that through the whole system but only that through the fraction of the system drained by the vein sampled. Therefore, in defining the steady state it is not enough to demonstrate that total flow is constant but it must be shown that flow: 
through that part of the system represented by $C_{V}(t)$ is constant.

\section{SUMMARY AND RECOMMENDATIONS}

Rate of tissue metabolism, uptake or release, can be measured by multiplying blood (or plasma, whichever is appropriate) flow by arteriovenous difference only if flow is constant, arterial concentration is constant, and rate of tissue metabolism is constant. A further restriction is that if venous concentration is not identical in all veins draining the tissue the venous concentration used must be weighted by the relative contribution to total outflow made by the bed drained by the vein sampled.

If flow varies with time the distribution of transit times through the system is apt to be unmeasurable and the system cannot be handled by equations that depend on the superposition principle; that is, the system is apt to become a nonlinear nonstationary system. During such changes in flow there does not appear to be any reliable way to interpret the data. It is clear only that an increase in flow will probably lead to an overestimate of tissue uptake and a decrease in flow to an underestimate. These considerations eliminate the possibility of using $\mathrm{A}-\mathrm{V}$ differences to measure metabolism when flow varies. As soon as flow has remained constant for a time equal to the longest transit time through the system, it can be regarded as satisfactory for use in estimation of metabolism. This time can be determined for each system by indicator dilution techniques. Phasic changes in flow about a stable mean can be regarded as constant provided the period is brief compared with mean transit time through the system.

With these restrictions in mind we assume in what follows that flow is constant. If either uptake or output is a function of time, the distribution of transit times through the system is not stationary but the system is probably satisfactorily represented as linear; that is, the transformations with respect to time of various inputs through the system are superposable. Because tissue metabolism is an event occurring within the system, the transit times from entrance to sites of metabolism or from sites of metabolism to exit are the ones we wish to measure but apparently can't at present. We can, however, plausibly relate the desired transit times to measurable transit times through the system. To find the metabolic rate at time $t$, clearly we need to use arterial concentration at some time before $t$ and venous concentration at some time after $t$. For linear changes in uptake or output we are able to make these temporal corrections in arterial and venous concentration by using the approximate mean transit time which can be estimated by indicator dilution methods. For a nonmetabolized substance, mean transit time is its volume of distribution, $V$, divided by the flow $F$ (plasma or blood water, in whichever it is dissolved) through the system. For most metabolites the appropriate volume of distribution is probably the total extracellular volume of the system. In the case of glucose, for example, mean transit time through the forearm of man in the basal state is about 10 minutes, which is a considerable correction on some time scales. The magnitude of the time correction also determines the frequency with which blood samples should be taken. It is probably prudent to sample at intervals no greater than the mean transit time unless arterial and venous concentrations are relatively stable or otherwise well defined.

If arterial concentration is also a function of time, the data are manageable if the function is well defined. This means that it must be measured often enough to define it. If the change in arterial concentration is linear with time, the correction is made by moving back in time to that arterial concentration, found or properly interpolated, that occurred one mean transit time unit earlier. Treatment of combinations of variations follows the same principle and is given in the body of this paper. The uncertainties are such that the investigator is urged wherever possible to design his experiments so that arterial concentration is held constant or so that he changes arterial concentration stepwise from one steady state to another. The time required to reach the steady state is not the mean transit time but the longest transit time through the system. For the case of glucose through the forearm of man in the basal state, the longest transit time is about 30 minutes.

In the introduction an operational definition of constant was promised. If the temporal cor- 
rections proposed here do not lead to a corrected $\mathrm{A}-\mathrm{V}$ difference that differs from the simultaneous A-V difference at time $t$ by more than the experimental error of measurement of an A-V difference, it is probably safe to regard the functions as constant.

The effect of the non-steady state on simultaneous A-V differences means that a solitary $\mathrm{A}-\mathrm{V}$ difference is uninterpretable. The immediate history of the system must be known. A single pair of A-V differences determined simultaneously on two substances passing through the same bed is also not properly useful for comparing the relative metabolism of the two substances unless it is known that the distributions of their transit times are identical.

\section{REFERENCES}

1. Cori, C. F., Fisher, R. E., and Cori, G. T. The effect of epinephrine on arterial and venous plasma sugar and blood flow in dogs and cats. Amer. J. Physiol. 1935, 114, 53.

2. Visscher, M. B., and Johnson, J. A. The Fick principle: Analysis of potential errors in its conventional application. J. appl. Physiol. 1953, 5, 635.

3. Fishman, A. P., McClement, J., Himmelstein, A., and Cournand, A. Effects of acute anoxia on the circulation and respiration in patients with chronic pulmonary disease studied during the "steady state." J. clin. Invest. 1952, 31, 770.
4. Fick, A. Ueber die Messung des Blutquantums in den Herzventrikeln. S.-B. phys.-med. Ges. Würzburg 1870, p. 16.

5. Grant, R. T. Observations on the blood circulation in voluntary muscle in man. Clin. Sci. 1938, 3, 157.

6. Stephenson, J. L. Theory of the measurement of blood flow by the dilution of an indicator. Bull. math. Biophys. 1948, 10, 117.

7. Meier, P., and Zierler, K. L. On the theory of the indicator-dilution method for measurement of blood flow and volume. J. appl. Physiol. 1954, 6, 731.

8. Zierler, K. L. A simplified explanation of the theory of indicator-dilution for measurement of fluid flow and volume and other distributive phenomena. Bull. Johns Hopk. Hosp. 1958, 103, 199.

9. Zierler, K. L. Circulation times and the theory of indicator-dilution methods for determining blood flow and volume in Handbook of Physiology, vol. I., sect. 2, Circulation. American Physiological Society. In press.

10. Sherman, H. On the theory of indicator-dilution methods under varying blood-flow conditions. Bull. math. Biophys. 1960, 22, 417.

11. Zadeh, L. A. Frequency analysis of variable networks. Proc. IRE 1950, 38, 291.

12. Andres, R., Cader, G., and Zierler, K. L. The quantitatively minor role of carbohydrate in oxidative metabolism by skeletal muscle in intact man in the basal state. Measurements of oxygen and glucose uptake and carbon dioxide and lactate production in the forearm. J. clin. Invest. 1956, 35, 671.

\section{CORRECTION}

On page 2000 of the article entitled "Studies of the BloodCerebrospinal Fluid Barrier to Cortisol in the Dog" by Nicholas P. Christy and Robert A. Fishman (J. clin. Invest. 1961, 40, 1997), lines 9 and 10 in the right-hand column should read: "Samples of plasma and CSF were taken at hourly intervals. Constant levels of cortisol in plasma and CSF were attained after 4 hours and maintained for the succeeding 5 hours." 